Annali di Matematica pura ed applicata

(IV), Vol. CLXXVI (1999), pp. 191-207

\title{
Chaos in Singular Systems $(*)$.
}

\author{
SANDRA Chiarelli
}

\begin{abstract}
In this paper we consider singular systems of differential equations and we show that, under right conditions, the Poincaré map associated to those systems, and not just a suitable iterate, behaves chaotically. We use the notion of exponential dichotomies to prove the existence of a transverse homoclinic orbit of our system and after use the shadow lemma to show that the Poincare map associated to its topologically conjugate to the Bernouilli shift on a set of two symbols.
\end{abstract}

\section{1. - Introduction.}

Let $F$ be a diffeomorphism on $\mathrm{R}^{n}$. It is known [8,9], that, if $F$ has a transverse homoclinic point, there exists an invariant Cantor-like set on which a suitable iterate $F$ behaves like the Bernoulli shift on a finite number of symbols. On the other hand, when $F$ is the Poincaré map associated to a nonlinear oscillator $\dot{x}=f(t, x)$, the existence of a transverse homoclinic point of $F$ is equivalent to the existence of a "Transverse homoclinic orbit" $\gamma(t)$ of $\dot{x}=f(t, x)$, homoclinic to a hyperbolic periodic orbit $x_{0}(t)$, that is a solution $\gamma(t)$ such that

$$
\lim _{t \rightarrow \pm \infty}\left|\gamma(t)-x_{0}(t)\right|=0
$$

and the variational system

$$
\dot{x}=f_{x}(t, \gamma(t)) x
$$

has an exponential dichotomy on $\mathbb{R}[8]$.

However, recognizing the existence of such a kind of orbits is not always an easy task, and it is often reduced, when the system is

$$
\dot{x}=f(x)+\varepsilon g(t, x, \varepsilon), \quad x \in \mathbb{R}^{n}, \quad g(t+T, x, \varepsilon)=g(t, x, \varepsilon)
$$

and $\dot{x}=f(x)$ has a (necessarily non-transverse) homoclinic orbit $\gamma_{0}(t)$, to the study of a Melnikov function $M(\alpha)$. Actually, it is known $[3,6,8]$ that, if $M(\alpha)$ has a simple zero at

(*) Entrata in Redazione il 3 aprile 1997 e, in versione riveduta, il 30 ottobre 1997.

Indirizzo dell'A.: Via Bagnaturo 76, 67039, Sulmona, L'Aquila. Tel. 0864-251309 (Cell. 0347/3712671). 
$\alpha=\alpha_{0}$ and $\gamma_{0}(t)$ satisfies a suitable non degenerateness condition, the Poincare Map associated to (1) has a transverse homoclinic orbit with associated chaotic behavior. Both this property of the Melnikov function and Smale theorem were first proved by geometrical arguments. Starting from [3] analytical methods have been applied to this kind of problems and Palmer [8] in 1984 gave an analytical proof of Smale theorem making use of a kind of «Shadow lemma" for diffeomorphism with a hyperbolic invariant set.

Recently, investigators have addressed their attentions to singular systems of differential equations like:

$$
\varepsilon \dot{x}=f(x)+\varepsilon^{p} g(t, x, \varepsilon)
$$

where $x \in \mathrm{R}^{n}, g(t+1 T, x, \varepsilon), p>0$.

It is assumed that $f$ and $g$ are sufficiently smooth $\left(\mathfrak{C}^{2}\right)$ in their arguments and that the "Boundary layer" system $\dot{x}=f(x)$ has a homoclinic orbit $\gamma_{0}(t)$ such that the variational system $\dot{x}=f^{\prime}\left(\gamma_{0}(t)\right) x$ has the unique bounded solution $\dot{\gamma}_{0}(t)$, up to a multiplictive constant (non degenerateness). As an example we mention the Duffing equation

$$
\ddot{z}+\alpha^{2} f_{0}(z)=h(t), \quad z \in \mathrm{R}, \quad h(t)=h(t+T)
$$

where $\alpha \gg 1$ is a sufficiently large real parameter.

Setting $\varepsilon=\alpha^{-1}>0, z=x_{1}, \varepsilon \dot{z}=x_{2}$, equation (3) reads:

$$
\varepsilon \dot{x}_{1}=x_{2}, \quad \varepsilon \dot{x}_{2}=-f_{0}\left(x_{1}\right)+\varepsilon^{2} h(t)
$$

and we fall in the class of singular system as in (2).

Note that the "boundary layer" equation is, in this case, $\ddot{z}=f_{0}(z)=0$ and our conditions are that this equation has a homoclinic orbit to a hyperbolic equilibrium point, the non-degenerateness hypothesis being automatically satisfied because the equation is of the 2nd order in R. It is known [2] that, if a certain Melnikov function, associated to (3), has a simple zero at some point, equation (3) has a transverse homoclinic orbit.

This fact, together with Smale theorem implies chaotic behaviour of an iterate of the associated Poincaré Map. However, recently, BATTELLI and FECKMAN [1] have shown that, under the same conditions, there exists $\varepsilon_{0}>0$ such that equation (2) has periodic solutions of period $m T$, for any $m \in \mathbb{N}$ and $0<\varepsilon<\varepsilon_{0}$.

This fact lead to think that the Poincaré map itself behaves chaotically and not just a suitable iterate.

The purpose of this paper is to show that the above conjecture is in fact true. To this end we will follow the approach in [8] and use the notion of exponential dichotomies and the shadow lemma as stated in [8]. Let us briefly explain the plan of this work.

In Section 2 we recall the notion of exponential dichotomies and prove the existence of a transverse heteroclinic orbit of (2). This kind of result was already proved in [2] where they used scales of Banach-spaces, in order to prove the smoothness with respect to $\varepsilon$ of a «bifurcation function" from which the Melnikov condition is derived.

Our method of proof, instead, is inspired by [1] (where, however the proof of transversality was missed) and does not need to show the smoothness in $\varepsilon$. This fact has also the consequence that the method can be applied even when $p>0$ is real. In Section 3 we recall the shadow-lemma proved in [8] and show the main result of this paper stat- 
ing that the Poincaré Map of system (2) is homeomorphic to the Bernoulli shift on a set of two symbols.

\section{2. - Existence of transverse homoclinic orbits.}

Let $A(t)$ a $n \times n$ bounded and continuous matrix on an interval $I \subseteq \mathrm{R}$. We will say that the linear system on $\mathrm{R}^{n}$

$$
\dot{x}(t)=A(t) x(t), \quad x \in \mathbb{R}^{n},
$$

has an exponential dichotomy on $I$, if there exists $K \geqslant 1, \delta>0$ and a projection $P$ such hat the fundamental matrix $X(t)$ of $(4)$, which satisfies $X(0)=\mathbb{I}$, satisfies

$$
\begin{aligned}
& \left\|X(t) P X^{-1}(s)\right\| \leqslant K e^{-\delta(t-s)}, \quad \forall s, t \in I, \quad s \leqslant t, \\
& \left\|X(t)(I-P) X^{-1}(s)\right\| \leqslant K e^{+\delta(t-s)}, \quad \forall s \geqslant t, \quad s, t \in I .
\end{aligned}
$$
$t \in I$.

When (4) has an exponential dichotomy on $I \subseteq \mathrm{R}$ we set $\mathscr{P}(t)=X(t) P X^{-1}(t)$,

The projections $P, \mathscr{P}(t)$ are not uniquely determined however, if $I=\mathrm{R}_{+}$the range of $\mathscr{P}(0), \mathscr{R} \mathscr{P}(0)$, must be the stable subspace

$$
\left\{\xi \in \mathrm{R}^{n}: \sup _{t \geqslant 0}\left|X(t) X^{-1}(0) \xi\right|<\infty\right\}
$$

but the kernel may be any complementary subspace; while, if $I=\mathbf{R}_{\text {- }}$ the kernel of $\mathscr{P}(0)$, $\Re \mathscr{P}(0)$, must be the unstable subspace

$$
\left\{\xi \in \mathbb{R}^{n}: \sup _{t \leqslant 0}\left|X(t) X^{-1}(0) \xi\right|<\infty\right\}
$$

but the range may be any complementary subspace $[4,8]$.

As a consequence, if (4) has an exponential dichotomy on $\mathbb{R}$, both $\Re P$ and $\Re P$ are uniquely determined and hence so are $P, \mathcal{P}(t)$.

Let $C_{b}^{0}\left(\mathbf{R}_{+}, \mathbf{R}^{n}\right)$ the Banach space of all $\mathbf{R}^{n}$-valued functions which are continuous in $\mathbf{R}_{+}$and bounded, $C_{b}^{0}\left(R_{-}, \mathbf{R}^{n}\right)$ the Banach space of all $\mathbf{R}^{n}$-valued functions which are continuous in $R_{-}$and bounded.

The following lemma [8] is of basic importance:

Lemma 2.1. - Let (4) have an exponential dichotomy on $\mathbb{R}_{+}$with constants $k \geqslant 1$, $\delta>0$ and projection $P_{+}$.

Then for any $h \in C_{b}^{0}\left(R_{+}, \mathbb{R}^{n}\right), \forall \xi \in \mathscr{R} P_{+}$, there exists a unique solution $x(t)$ of the system

$$
\left\{\begin{array}{l}
\dot{x}(t)=A(t) x(t)+h(t), \\
P_{+} x(0)=\xi,
\end{array}\right.
$$


$x(t) \in C_{b}^{0}\left[\mathrm{R}_{+}\right]$given by

$$
x(t)=X(t) \xi+\int_{0}^{t} X(t) P_{+} X^{-1}(s) h(s) d s-\int_{t}^{\infty} X(t) Q_{+} X^{-1}(s) h(s) d s
$$

where $Q_{+}=\mathbb{I}-P_{+}$.

Moreover $\|x\| \leqslant \mathfrak{C}\left({ }^{\circ} h^{\circ}+|\xi|\right)$.

REMARK. - We have a similar result when the dichotomy is on $R_{-}$. In this case the system

$$
\left\{\begin{array}{l}
\dot{x}(t)=A(t) x(t)+h(t), \quad \eta \in \Re P_{-}, \\
Q_{-} x(0)=\eta,
\end{array}\right.
$$

has the unique solution bounded on $\mathbb{R}_{-}$

$$
x(t)=X(t) \eta+\int_{-\infty}^{t} X(t) P_{-} X^{-1}(s) h(s) d s-\int_{t}^{0} X(t)\left[\mathrm{I}-P_{-}\right] X^{-1}(s) h(s) d s
$$

with $\|x(t)\| \leqslant \mathcal{C}\left({ }^{\circ} h^{\circ}+|\eta|\right)$.

In order to apply the above lemma and remark to our problem, we simply need to note the following facts. As $p$ is a hyperbolic equilibrium point, the linear system $\dot{x}=$ $=f^{\prime}(p) x$ has an exponential dichotomy on $\mathrm{R}$ with projection $P$ that can be chosen to commute with $X(t)$ (hence $\mathscr{P}(t)=P, \forall t \in \mathrm{R}$ ).

Now, $\gamma_{0}(t)$ is homoclinic to $p$, thus it follows from the roughness of exponential dichotomies [4] that the linear system $\dot{x}=f^{\prime}\left(\gamma_{0}(t)\right) x$ has an exponential dichotomy on $\mathbf{R}_{+}$ and $\mathrm{R}_{-}$, with constants $k, \delta$ and projection $P_{+}, P_{-}$, which satisfy

$\operatorname{dim} R P_{+}=\operatorname{dim} R P=$ number of eigenvalues of $f^{\prime}(p)$ with negative real part counted with their multiplicities,

$\operatorname{dim} \Re P_{-}=\operatorname{dim} \Re P=$ number of eigenvalues of $f^{\prime}(p)$ with positive real part counted with their multiplicities.

Moreover [8] we have

$$
\begin{array}{lll}
\left|P_{+}(t)-P\right| \rightarrow 0 & \text { as } & t \rightarrow+\infty, \\
\left|P_{-}(t)-P\right| \rightarrow 0 & \text { as } & t \rightarrow-\infty .
\end{array}
$$

Finally, as $\gamma_{0}(t)$ is homoclinic, the system $\dot{x}=f^{\prime}\left(\gamma_{0}(t)\right) x$ and its adjoint $\dot{x}=-$ $-f^{\prime}\left(\gamma_{0}(t)\right)^{*} \cdot x$ (here and in the following * means transpose) have the same number of (independent) bounded solutions. So, as we assume that the space of bounded solutions of the variational system $\dot{x}=f^{\prime}\left(\gamma_{0}(t)\right)$ is spanned by $\dot{\gamma}_{0}(t)$, there exists a unique, up to a multiplicative constant, non-zero bounded solution $\psi(t)$ of $\dot{x}=-f^{\prime}\left(\gamma_{0}(t)\right)^{*} \cdot x$.

Without loss of generality we can also assume that $\psi=\psi(0)$ is such that $|\psi(0)|=$ $=1$. We are now able to prove the main result of this section: 
THEOREM 2.1. - Given the singular system

$$
\varepsilon \dot{x}=f(x)+\varepsilon g(t, x, \varepsilon)
$$

$f(x), g(t, x, \varepsilon)$ being regular $\left(\mathrm{e}^{r}, r \geqslant 1\right)$ and bounded in their arguments and $g, T$-periodic in $t$, suppose that the boundary layer problem

$$
\dot{x}=f(x)
$$

has a solution $\gamma_{0}(t)$ homoclinic to a hyperbolic equilibrium point $p$.

Furthermore, assume that the variational system

$$
\dot{x}=\frac{\partial f}{\partial x}\left(\gamma_{0}(t)\right) \cdot x
$$

has the unique bounded solution $\dot{\gamma}(t)$.

Then, if there exists $\alpha_{0}$ such that

$$
\int_{-\infty}^{+\infty} \psi^{*}(t) g\left(\alpha_{0}, \gamma_{0}(t), 0\right) d t=0
$$

and

$$
\int_{-\infty}^{+\infty} \psi^{*}(t) g\left(\alpha_{0}, \gamma_{0}(t), 0\right) d t \neq 0,
$$

there exists a unique $\alpha(\varepsilon)$ with, $\alpha(0)=\alpha_{0}$ such that the system

$$
\varepsilon \dot{x}=f(x)+\varepsilon g(t, x, \varepsilon)
$$

has a unique solution $\gamma(t, \varepsilon)$ satisfying

$$
\sup _{t \in \mathbb{R}}\left|\gamma(t, \varepsilon)-\gamma_{0}\left(\varepsilon^{-1}(t-\alpha(\varepsilon))\right)\right|=O(\varepsilon) .
$$

Moreover the variational system

$$
\varepsilon \dot{x}=\left[f_{x}(\gamma(t, \varepsilon))+\varepsilon g_{x}(t, \gamma(t, \varepsilon), \varepsilon)\right] \cdot x
$$

has an exponential dichotomy on $\mathrm{R}$ with constants $k, \delta / \varepsilon$.

Proof. - The proof of existence of $\gamma(t, \varepsilon)$ is known. However we will give it here for completeness and mainly because we need it to show the "transversality condition".

Put $u(t)=x(\varepsilon t+\alpha)-\gamma_{0}(t)$. Finding a solution of (5) is equivalent to the study of $\dot{u}(t)-A(t) u(t)=f\left(u(t)+\gamma_{0}(t)\right)-f\left(\gamma_{0}(t)-A(t) u(t)+\varepsilon g\left(\varepsilon t+\alpha, u(t)+\gamma_{0}(t), \varepsilon\right)\right.$ 
or

$$
\dot{u}(t)=A(t) u(t)+h(t, u, \alpha, \varepsilon)
$$

with

$$
\begin{gathered}
A(t)=f^{\prime}\left(\gamma_{0}(t)\right) \\
h(t, u, \alpha, \varepsilon)=f\left(u(t)+\gamma_{0}(t)\right)-f\left(\gamma_{0}(t)-A(t) u(t)+\varepsilon g\left(\varepsilon t+\alpha, u(t)+\gamma_{0}(t), \varepsilon\right) .\right.
\end{gathered}
$$

Applying Lemma 2.1 and the contraction mapping principle we have: for all $\xi \in \mathscr{R} P_{+}$, such that $\langle\xi, \dot{\gamma}(0)\rangle=0$, and $\eta \in \Re P_{-}$there exist unique bounded solutions $u_{+}$of

$$
\left\{\begin{array} { l } 
{ \dot { u } ( t ) - A ( t ) u ( t ) = h ( t , u , \alpha , \varepsilon ) , } \\
{ P _ { + } u ( 0 ) = \xi , \quad t \geqslant 0 , }
\end{array} \text { and } u _ { - } \text { of } \quad \left\{\begin{array}{l}
\dot{u}(t)-A(t) u(t)=h(t, u, \alpha, \varepsilon), \\
Q_{-} u(0)=\eta, t \leqslant 0,
\end{array}\right.\right.
$$

satisfying:

$$
\begin{gathered}
\left\|u_{+}(\cdot, \xi, \alpha, \varepsilon)\right\| \leqslant 2 K\left\{|\xi|+2 \delta^{-1} M|\varepsilon|\right\}, \\
\left\|u_{-}(\cdot, \eta, \alpha, \varepsilon)\right\| \leqslant 2 K\left\{|\eta|+2 \delta^{-1} M|\varepsilon|\right\}, \\
\left\|\frac{\partial u_{+}}{\partial \xi}(\cdot, \xi, \alpha, \varepsilon)\right\| \leqslant \mathcal{C}, \quad\left\|\frac{\partial u_{-}}{\partial \eta}(\cdot, \eta, \alpha, \varepsilon)\right\| \leqslant \mathcal{C}, \\
\left\|\frac{\partial u_{ \pm}}{\partial \alpha}(\cdot, \xi, \eta, \alpha, \varepsilon)\right\| \leqslant \mathcal{C}|\varepsilon|,
\end{gathered}
$$

because of the definition of $h$ and the conditions on $f$ and $g$ (see also [1]).

Moreover if $u(t)$ is a bounded solution of (6), $u(t)$ satisfies both the first and the second system with $\xi=P_{+} u(0)$ and $\eta=Q_{-} u(0)$.

So, the problem of existence of bounded solutions of (6) is equivalent to solving

$$
u_{+}(0)-u_{-}(0)=0 \text {. }
$$

We have

$$
\begin{aligned}
& u_{+}(0)-u_{-}(0)=X(0) \xi-\int_{0}^{\infty} X(0) Q_{+} X^{-1}(s) h\left(s, u_{+}(s), \alpha, \varepsilon\right) d s-X(0) \eta+ \\
& -\int_{-\infty}^{0} X(0) P_{-} X^{-1}(s) h\left(s, u_{-}(s), \alpha, \varepsilon\right) d s= \\
& =\xi-\eta-\int_{0}^{\infty} Q_{+} X^{-1}(s) h\left(s, u_{+}(s), \alpha, \varepsilon\right) d s+
\end{aligned}
$$




$$
\begin{aligned}
& -\int_{-\infty}^{0} P_{-} X^{-1}(s) h\left(s, u_{-}(s), \alpha, \varepsilon\right) d s u_{+}(0)-u_{-}(0)=0 \Leftrightarrow \xi-\eta= \\
& =\int_{0}^{\infty} Q_{+} X^{-1}(s) h\left(s, u_{+}(s), \alpha, \varepsilon\right) d s+\int_{-\infty}^{0} P_{-} X^{-1}(s) h\left(s, u_{-}(s), \alpha, \varepsilon\right) d s=H(\xi, \eta, \alpha, \varepsilon) .
\end{aligned}
$$
have

Notice that if $\xi \in \mathscr{R} P_{+}$and $\eta \in \mathscr{N} P_{-}$are such that $\xi-\eta=H(\xi, \eta, \alpha$, $\varepsilon$ ), we

$$
\psi^{*} \cdot H=0
$$

because $\xi-\eta \in\left[\Re P_{+}+\mathscr{T} P_{-}\right]=\{\psi\}^{\perp}$, then we can study

$$
\left\{\begin{array}{l}
\xi-\eta=H(\xi, \eta, \alpha, \varepsilon)+\psi^{*} \cdot H(\xi, \eta, \alpha, \varepsilon) \psi, \\
\psi^{*} \cdot H(\xi, \eta, \alpha, \varepsilon)=0
\end{array}\right.
$$

(this procedure is called the "Lyapunov-Schmidt procedure»).

It follows from the definition of $H(\xi, \eta, \alpha, \varepsilon)$ that $|H(\xi, \eta, \alpha, \varepsilon)| \leqslant 2 K \delta^{-1}\|h\|$ and because of $h(t, u, \alpha, \varepsilon)=f\left(u(t)+\gamma_{0}(t)\right)-f\left(\gamma_{0}(t)\right)-A(t) u(t)+\varepsilon g(\varepsilon t+\alpha, u(t)+$ $\left.+\gamma_{0}(t), \varepsilon\right)$ we get

$$
|H(\xi, \eta, \alpha, \varepsilon)| \leqslant \mathcal{C}(|\xi|+|\eta|+|\varepsilon|)
$$

uniformly with respect to $\alpha$.

In the same way we get

$$
\begin{gathered}
\left|\frac{\partial H}{\partial \xi}(\xi, \eta, \alpha, \varepsilon)\right| \leqslant \mathcal{C}(|\xi|+|\eta|+|\varepsilon|), \\
\left|\frac{\partial H}{\partial \eta}(\xi, \eta, \alpha, \varepsilon)\right| \leqslant \mathcal{C}(|\xi|+|\eta|+|\varepsilon|), \quad\left|\frac{\partial H}{\partial \alpha}(\xi, \eta, \alpha, \varepsilon)\right| \leqslant \mathfrak{e}|\varepsilon| .
\end{gathered}
$$

Hence, for $\varepsilon$ sufficiently small, the uniform contraction theorem gives the existence of a unique pair of functions $\xi(\alpha, \varepsilon), \eta(\alpha, \varepsilon)$ such that $\xi(\alpha, \varepsilon) \leqslant \mathcal{C}|\varepsilon|, \eta(\alpha, \varepsilon) \leqslant \mathcal{C}|\varepsilon|$ satisfying the first equation of (7).

Moreover it follows from (8), (9), (10) that

$$
\left|\frac{\partial \xi}{\partial \alpha}(\alpha, \varepsilon)\right| \leqslant \mathcal{C}|\varepsilon|, \quad\left|\frac{\partial \eta}{\partial \alpha}(\alpha, \varepsilon)\right| \leqslant \mathcal{C}|\varepsilon|,
$$

$\mathcal{C}$ being independent of $\alpha$.

The problem of finding solutions $\gamma(t, \varepsilon)$ "near» $\gamma_{0}(t)$ is then equivalent to solving the bifurcation equation $G(\alpha, \varepsilon)=0$ where

$$
G(\alpha, \varepsilon)=\psi^{*} \cdot H(\xi(\alpha, \varepsilon), \eta(\alpha, \varepsilon), \alpha, \varepsilon) .
$$

Define now $\widetilde{G}(\alpha, \varepsilon)=\varepsilon^{-1} G(\alpha, \varepsilon)$. 
As

$$
\psi^{*} Q_{+} X^{-1}(s)=\left[X^{-1}(s)^{*} Q_{+}^{*} \psi\right]^{*}=\left\{X^{-1}(s)^{*}\left(I-P_{+}^{*}\right) \psi\right\}^{*}=\left\{X^{-1}(s)^{*} \psi\right\}^{*}
$$

and

$$
\psi^{*} P_{-} X^{-1}(s)=\left[X^{-1}(s)^{*} P_{-}^{*} \psi\right]^{*}=\left\{X^{-1}(s)^{*} \psi\right\}^{*}
$$

we get

$$
\begin{aligned}
& \tilde{G}(\alpha, \varepsilon)=\varepsilon^{-1} \int_{-\infty}^{+\infty} \psi^{*}(t) h\left(t, u_{ \pm}(t, \xi(\alpha, \varepsilon), \eta(\alpha, \varepsilon), \alpha, \varepsilon)\right) d t= \\
& =\varepsilon^{-1} \int_{-\infty}^{+\infty} \psi^{*}(t)\left\{f\left(u_{ \pm}+\gamma_{0}(t)\right)-f\left(\gamma_{0}(t)\right)-A(t) u_{ \pm}(t)+\varepsilon g\left(\varepsilon t+\alpha, u_{ \pm}+\gamma_{0}(t), \varepsilon\right)\right\} d t .
\end{aligned}
$$

As

$$
f\left(u_{ \pm}+\gamma_{0}(t)\right)-f\left(\gamma_{0}(t)\right)-A(t) u(t)=O\left(\left\|u_{ \pm}\right\|_{b}^{2}\right)=O\left(|\varepsilon|^{2}\right)
$$

we have

$$
\widetilde{G}(\alpha, \varepsilon)=\int_{-\infty}^{+\infty} \psi^{*}(t) g\left(\varepsilon t+\alpha, u_{ \pm}(t)+\gamma_{0}(t), \varepsilon\right) d t+O(|\varepsilon|)
$$

which tends to $M(\alpha)=\int_{-\infty}^{+\infty} \psi^{*}(t) g\left(\alpha, \gamma_{0}(t), 0\right) d t$ as $\varepsilon \rightarrow 0^{+}$.

Moreover this convergence holds together with the derivatives with respect to $\alpha$. In fact for example

$$
\begin{aligned}
\int_{-\infty}^{+\infty} \mid \psi^{*}(t)\left\{\left[f_{x}\left(u_{ \pm}(t, \alpha)+\gamma_{0}(t)\right)-\right.\right. & \left.A(t)] \frac{\partial u_{ \pm}}{\partial \alpha}(t, \alpha)\right\} \mid d t \leqslant \\
& \leqslant \int_{-\infty}^{+\infty} k e^{-\delta|t|}\left\{N^{0} u_{ \pm}(\cdot, \alpha, \varepsilon)^{0} \cdot\left\|\frac{\partial u_{ \pm}}{\partial \alpha}(\cdot, \alpha, \varepsilon)\right\|\right\} d t
\end{aligned}
$$

But

$$
\left\|u_{ \pm}(\cdot, \alpha, \varepsilon)\right\|=O(|\varepsilon|)
$$

and

$$
\left\|\frac{\partial u_{ \pm}}{\partial \alpha}(\cdot, \alpha, \varepsilon)\right\|=O(|\varepsilon|)
$$


so

$$
\int_{-\infty}^{+\infty}\left|\psi^{*}(t)\left\{\left[f_{x}\left(u_{ \pm}(t, \alpha)+\gamma_{0}(t)\right)-A(t)\right] \frac{\partial u_{ \pm}}{\partial \alpha}(t, \alpha)\right\}\right| d t=O\left(|\varepsilon|^{2}\right) .
$$

In the same way we see that

$$
\int_{-\infty}^{+\infty} \psi^{*}(t) \cdot \frac{\partial g}{\partial x}\left(\varepsilon t+\alpha, u_{ \pm}(t)+\gamma_{0}(t), \varepsilon\right) \cdot \frac{\partial u_{ \pm}}{\partial \alpha}(t, \alpha) d t=O(|\varepsilon|) .
$$

$M(\alpha)$ is our «Melnikov function».

Now, we known there exists $\alpha_{0}$ such that $M\left(\alpha_{0}\right)=0 \neq M^{\prime}\left(\alpha_{0}\right)$, then form the uniform contraction theorem it follows that there exists a unique $\alpha(\varepsilon)$ such that $\widetilde{G}(\alpha(\varepsilon), \varepsilon)=0$ with $0<\varepsilon<\varepsilon_{0} \ll 1$.

So there exists a unique solution $u(t, \varepsilon)$ of the system

$$
\dot{u}(t)=A(t) u(t)+h(t, u, \alpha(\varepsilon), \varepsilon) .
$$

Changing $t$ with $\varepsilon t+\alpha$ and setting $\gamma(t, \varepsilon)=u\left(\varepsilon^{-1}(t-\alpha(\varepsilon), \varepsilon)\right)+\gamma_{0}\left(\varepsilon^{-1}(t-\right.$ $-\alpha(\varepsilon)))$, we get

$$
\sup _{\tau \in \mathbb{R}}\left|\gamma(t, \varepsilon)-\gamma_{0}\left(\varepsilon^{-1}(t-\alpha(\varepsilon))\right)\right| \leqslant C|\varepsilon|
$$

To prove that $\gamma(t, \varepsilon)$ is a traverse solution we need the following theorem about the local invertibility for operators [5].

Theorem 2.2. - Let $X, Y$ be Banach spaces.

Consider a $C^{r}$-smooth map $F: X \rightarrow Y$ satisfying $F(0)=0$ and assume $D F(0)$ is Fredholm with index zero.

If there exist a neighborhood $U \subseteq X$ of 0 and real numbers $k>0, \delta>0$ such that for any linear bounded mapping $B: X \rightarrow Y,\|B\| \leqslant k$, the perturbations $\varepsilon B+F, 0 \leqslant \varepsilon<\delta$, has the only zero point 0 in $U$, then $D F(0)$ is invertible.

Notice that putting $y(t)=x(\varepsilon t+\alpha(\varepsilon))$, the system, (5) is equivalent to system

$$
\dot{y}=f(y)+\varepsilon g(\varepsilon t+\alpha(\varepsilon), y, \varepsilon) \text {. }
$$

Furthermore if the system

$$
\dot{y}=\left[f_{x}\left(u(t, \varepsilon)+\gamma_{0}(t)\right)+\varepsilon g_{x}\left(\varepsilon t+\alpha, u(t, \varepsilon)+\gamma_{0}(t), \varepsilon\right)\right] \cdot y
$$

has an exponential dichotomy with constants $k, \delta$ then the system $\varepsilon \dot{x}\left[f_{x}(\gamma(t, \varepsilon))+\right.$ $\left.+\varepsilon g_{x}(t, \gamma(t, \varepsilon), \varepsilon)\right] \cdot x$ has an exponential dichotomy with constants $k$ and $\delta / \varepsilon$.

So, we may study the system (12).

Consider the map

$$
\mathscr{F}_{\varepsilon}: C_{b}^{1}\left[\mathrm{R}, \mathrm{R}^{n}\right] \rightarrow C_{b}^{0}\left[\mathrm{R}, \mathbf{R}^{n}\right]
$$


such that

$$
\mathscr{F}_{\varepsilon}(y)=\dot{y}-f(y)-\varepsilon g(\varepsilon t+\alpha, y, \varepsilon) .
$$

As we have just shown there exists a unique $y(t, \varepsilon)=y_{\varepsilon}$ where $\mathscr{F}_{\varepsilon}\left(y_{\varepsilon}\right)=0$.

Let $\varepsilon_{0} \neq 0$. Let us define $\widetilde{\mathfrak{F}}_{\varepsilon}=\mathscr{F}_{\varepsilon}(y)+\varepsilon \sigma L\left(y-y_{\varepsilon_{0}}\right)$ such that $L: C_{b}^{1} \rightarrow C_{b}^{0}$ is a linear operator with $\|L\| \leqslant 1$ and $|\sigma|<\sigma_{0} \ll 1$.

Solving the following systems, as we did in the previous part of this theorem,

$$
\begin{aligned}
& \left\{\begin{array}{l}
\dot{y}-A(t) y-f(y)-\varepsilon g(\varepsilon t+\alpha, y, \varepsilon)+\varepsilon \sigma L\left(y-y_{\varepsilon_{0}}\right)+A(t) y=0, \\
P_{+} y(0)=\xi,
\end{array}\right. \\
& \left\{\begin{array}{l}
\dot{y}-A(t) y-f(y)-\varepsilon g(\varepsilon t+\alpha, y, \varepsilon)+\varepsilon \sigma L\left(y-y_{\varepsilon_{0}}\right)+A(t) y=0, \\
\left(\mathbb{1}-P_{-}\right) y(0)=\eta,
\end{array}\right.
\end{aligned}
$$

we have two bounded solutions, $y_{+}(t)$ and $y_{-}(t)$ respectively.

The same method as above shows, then, that for any $\sigma,|\sigma|<\sigma_{0} \ll 1, \tilde{F}_{\varepsilon}(y)=0$, $\varepsilon \neq 0$ has a unique solution $\tilde{y}_{\varepsilon}$ "near" to $y_{\varepsilon_{0}}$ uniformly in $\sigma$.

However $\tilde{F}_{\varepsilon_{0}}\left(y_{\varepsilon_{0}}\right)=\mathscr{F}_{\varepsilon_{0}}\left(y_{\varepsilon_{0}}\right)+\varepsilon_{0} \sigma L\left(y_{\varepsilon_{0}}-y_{\varepsilon_{0}}\right)=0$, so the unique solution of the equation $\tilde{\mathcal{F}}_{\varepsilon_{0}}(y)=0$ is $y_{\varepsilon_{0}}$. As $D \mathcal{F}_{\varepsilon}\left(y_{\varepsilon}\right)$ is Fredholm with index zero we may apply the Theorem 2.2, concluding that $D F_{\varepsilon}\left(y_{\varepsilon_{0}}\right)$ is an invertible operator. Then for any $h \in$ $\in C_{b}^{0}\left[\mathrm{R}, \mathrm{R}^{n}\right]$ the differential equation $\dot{y}\left[f_{x}\left(u(t, \varepsilon)+\gamma_{0}(t)\right)+\varepsilon g_{x}(\varepsilon t+\alpha, u(t, \varepsilon)+\right.$ $\left.\left.+\gamma_{0}(t), \varepsilon\right)\right] \cdot y=h(t)$ has a unique bounded solution.

If $h(t) \equiv 0$ the system

$$
\dot{y}-\left[f_{x}\left(u(t, \varepsilon)+\gamma_{0}(t)\right)+\varepsilon g_{x}\left(\varepsilon t+\alpha, u(t, \varepsilon)+\gamma_{0}(t), \varepsilon\right)\right] \cdot y=0
$$

has only the trivial solution as bounded solution. As

$$
\left[f_{x}\left(u(t, \varepsilon)+\gamma_{0}(t)\right)+\varepsilon g_{x}\left(\varepsilon t+\alpha(\varepsilon), u(t, \varepsilon)+\gamma_{0}(t), \varepsilon\right)\right] \underset{\varepsilon \rightarrow 0^{+}}{\longrightarrow} f_{x}\left(\gamma_{0}(t)\right),
$$

it follows from the roughness of exponential dichotomies, that the system (13) has an exponential dichotomy on $\mathbf{R}_{+}$and on $\mathbf{R}_{-}$with projection $\widetilde{P}_{+}, \widetilde{P}_{-}$such that

$$
\operatorname{dim} \Re \widetilde{P}_{+}=\operatorname{dim} R P_{+}, \quad \operatorname{dim} \Re \widetilde{P}_{-}=\operatorname{dim} \Re P_{-} .
$$

As a consequence $\operatorname{dim} R \widetilde{P}_{+}+\operatorname{dim} \Re \widetilde{P}_{-}=n$ and $\Re \widetilde{P}_{+} \cap \Re \widetilde{P}_{-}=\{0\}$ because of (13) has only the trivial bounded solution.

Then $\mathrm{R}^{n}=\Re \widetilde{P}_{+} \oplus \Re \widetilde{P}_{-}$and so

$$
\dot{y}=\left[f_{x}\left(u(t, \varepsilon)+\gamma_{0}(t)\right)+\varepsilon g_{x}\left(\varepsilon t+\alpha(\varepsilon), u(t, \varepsilon)+\gamma_{0}(t), \varepsilon\right)\right] \cdot y
$$

has an exponential dichotomy on $(-\infty,+\infty)$.

Remark. - Syppose (3) satisfies the same conditions hold in Theorem 2.1, then there exists a unique $T$-periodic solution $x_{0}(t, \varepsilon)$ such that $\sup _{t \in \mathbb{R}}\left|x_{0}(t, \varepsilon)-p\right| \rightarrow 0$ as
$\varepsilon \rightarrow 0^{+}$ 
The proof of this result follows the same procedure of the Theorem 2.1, and indeed, it is easier than it because of the invertibility of the linear part, and $\gamma(t, \varepsilon)$ tends to $x_{0}(t, \varepsilon)$ because of the saddle-point property.

\section{3. - Chaos of the Poincré map.}

Let $f(t, x)$ be a vector function, defined, bounded and continuous in $\mathbf{R} \times \mathbf{R}^{n}$. Suppose also that the partial derivative $f_{x}(t, x)$ exists, $f_{x} \in C_{b}^{0}\left[\mathbb{R} \times \mathbf{R}^{n}, \mathbf{R}^{n}\right], f_{x}$ continuous in $x$, uniformly with respect to $t$. Let us recall the following result:

THEOREM 3.1 («Shadowing lemma»). - Suppose for ech integer $k$ the system

$$
\dot{x}=f(t, x)
$$

has a solution $w_{k}(t)$ defined on an interval $\left[t_{k-1}, t_{k}\right]$ such that

(i) the variational equation

$$
\dot{x}=f_{x}\left(t, w_{k}(t)\right) \cdot x
$$

has an exponential dichotomy on $\left[t_{k-1}, t_{k}\right]$, with projection matrix function $\mathscr{P}_{k}(t)$ and constants $K, \alpha$;

$$
\begin{aligned}
& \left|w_{k-1}\left(t_{k-1}\right)-w_{k}\left(t_{k-1}\right)\right| \leqslant \delta ; \\
& \left|\mathscr{P}_{k-1}\left(t_{k-1}\right)-\mathcal{P}_{k}\left(t_{k-1}\right)\right| \leqslant \delta ;
\end{aligned}
$$

$$
t_{k}-t_{k-1} \geqslant \tau \text {. }
$$

Then there exist positive constants $\mu_{0}, \tau_{0}$ and a function $\delta_{0}(\mu)$ such that if $\tau \geqslant \tau_{0}$, $0 \leqslant \mu \leqslant \mu_{0}$ and $\delta \leqslant \delta_{0}(\mu)$, system (14) has a unique solution $x(t)$, satisfying $\mid x(t)-$ $-w_{k}(t) \mid \leqslant \mu$ for $t_{k-1} \leqslant t \leqslant t_{k}$ and all $k$.

In Section 2 we showed that, given the singular system

$$
\varepsilon \dot{x}=f(x)+\varepsilon g(t, x, \varepsilon)
$$

$f(x), g(t, x, \varepsilon)$, being $C_{b}^{r}$-function in their arguments $(r \geqslant 2)$, and $g(t, x, \varepsilon)$ being $T$-periodic in $t$, with the same hypotheses of the Theorem 2.1, setting $y(t)=x(\varepsilon t+\alpha(\varepsilon))$, system

$$
\dot{y}=f(y)+\varepsilon g(\varepsilon t+\alpha(\varepsilon), y, \varepsilon),
$$

equivalent to the system (5) has a unique transversal homoclinic orbit $u(t)$ such that

$$
\sup _{t \in \mathbf{R}}\left|u(t)-\gamma_{0}(t)\right| \leqslant C|\varepsilon|
$$

where $u(t)=u(t, \varepsilon)=\gamma(\varepsilon t+\alpha(\varepsilon), \varepsilon)$.

Now we are able to show the following result: 
THEOREM 3.2. - For each $a=\left\{a_{k}\right\}_{k \in Z}$ system

$$
\varepsilon \dot{x}=f(x)+\varepsilon g(t, x, \varepsilon)
$$

has a unique solution $x_{a}(t), t \in \mathrm{R}$, satisfying

$$
\sup _{-T / 2+\alpha(\varepsilon)<t<T / 2+\alpha(\varepsilon)}\left|x_{a}\left(t+(2 k-1) \frac{T}{2}\right)-\gamma\left(t+a_{k} T, \varepsilon\right)\right|<\varrho
$$

with $\varrho$ sufficiently small.

Proof. - Putting $y(t)=x(\varepsilon t+\alpha(\varepsilon))$ we may study system (11) and look for a solution $y_{a}(t)$ satisfying

$$
\sup _{-T / 2 \varepsilon \leqslant t \leqslant T / 2 \varepsilon}\left|y_{a}\left(t+(2 k-1) \frac{T}{2 \varepsilon}\right)-u\left(t+a_{k} \frac{T}{\varepsilon}\right)\right|<\varrho .
$$

For $u(t)=\gamma(\varepsilon t+\alpha(\varepsilon), \varepsilon)$ and for $k \in \mathbb{Z}$, we define

$$
v_{k}(t)=u\left(t+a_{k} \frac{T}{\varepsilon}\right), \quad w_{k}(t)=v_{k}\left(t-(2 k-1) \frac{T}{2 \varepsilon}\right), \quad t_{k}=k \frac{T}{\varepsilon} .
$$

Then $w_{k}(t)=u\left(t-(2 k-1)(T / 2 \varepsilon)+a_{k}(T / \varepsilon)\right)$ satisfies

$$
\begin{aligned}
\dot{w}=f(w)+\varepsilon g\left[\varepsilon\left(t-k \frac{T}{\varepsilon}+\frac{T}{2 \varepsilon}+a_{k} \frac{T}{\varepsilon}\right)+\alpha(\varepsilon), w, \varepsilon\right]= & \\
= & f(w)+\varepsilon g\left(\varepsilon t+\frac{T}{2}+\alpha(\varepsilon), w, \varepsilon\right)
\end{aligned}
$$

and the variational equation

$$
\begin{aligned}
& \dot{w}=\left[f^{\prime}\left(w_{k}(t)\right)+\varepsilon g_{x}\left(\varepsilon t+\frac{T}{2}+\alpha(\varepsilon), w_{k}(t), \varepsilon\right)\right] w= \\
&=\left[f^{\prime}\left(u\left(t-(2 k-1) \frac{T}{2 \varepsilon}+a_{k} \frac{T}{\varepsilon}\right)\right)+\varepsilon g_{x}\left(\varepsilon\left(t+\frac{T}{2 \varepsilon}\right)+\right.\right. \\
&\left.\left.\quad+\alpha(\varepsilon), u\left(t-(2 k-1) \frac{T}{2 \varepsilon}+a_{k} \frac{T}{\varepsilon}\right), \varepsilon\right)\right] w
\end{aligned}
$$

has an exponential dichotomy on $\mathbf{R}$ and so on $\left[t_{k-1}, t_{k}\right]$ with projection matrix function $\mathscr{P}_{k}(t)$. 
In fact, writing $t$ in the place of $t-T / 2 \varepsilon+\left(k+a_{k}\right)(T / \varepsilon)$ and recalling that $g(t, x, \varepsilon)$ is $T$-periodic in $t$, we have

$$
\dot{w}\left[f^{\prime}(u(t))+\varepsilon g_{x}(\varepsilon t+\alpha(\varepsilon), u(t), \varepsilon)\right],
$$

which has an exponential dichotomy on $\mathrm{R}$.

So the first condition of the Theorem 3.1 is verified.

Now we prove (ii), (iii), (iv).

(ii)

$$
\begin{aligned}
& \left|w_{k-1}\left(t_{k-1}\right)-w_{k}\left(t_{k-1}\right)\right|= \\
= & \left|v_{k-1}\left(t_{k-1}-(2 k-3) \frac{T}{2 \varepsilon}\right)-v_{k}\left(t_{k-1}-(2 k-1) \frac{T}{2 \varepsilon}\right)\right|= \\
= & \left|u\left(k \frac{T}{\varepsilon}-\frac{T}{\varepsilon}-k \frac{T}{\varepsilon}+3 \frac{T}{2 \varepsilon}+a_{k-1} \frac{T}{\varepsilon}\right)-u\left(k \frac{T}{\varepsilon}-\frac{T}{\varepsilon}-k \frac{T}{\varepsilon}+\frac{T}{2 \varepsilon}+a_{k} \frac{T}{\varepsilon}\right)\right|= \\
= & \left|u\left(\left(\frac{1}{2}+a_{k-1}\right) \frac{T}{\varepsilon}\right)-u\left(\left(a_{k}-\frac{1}{2}\right) \frac{T}{\varepsilon}\right)\right| \leqslant \\
\leqslant & \left|u\left(\left(\frac{1}{2}+a_{k-1}\right) \frac{T}{\varepsilon}\right)-\gamma_{0}\left(\left(\frac{1}{2}+a_{k-1}\right) \frac{T}{\varepsilon}\right)\right|+ \\
+ & \left|\gamma_{0}\left(\left(\frac{1}{2}+a_{k-1}\right) \frac{T}{\varepsilon}\right)-\gamma_{0}\left(\left(a_{k}-\frac{1}{2}\right) \frac{T}{\varepsilon}\right)\right|+ \\
& +\left|u\left(\left(a_{k}-\frac{1}{2}\right) \frac{T}{\varepsilon}\right)-\gamma_{0}\left(\left(a_{k}-\frac{1}{2}\right) \frac{T}{\varepsilon}\right)\right| \ll 1 \quad \text { if } \varepsilon \ll 1
\end{aligned}
$$

in fact

$$
\sup _{\mathbf{R}}\left|u(t)-\gamma_{0}(t)\right| \underset{\varepsilon \rightarrow 0^{+}}{\longrightarrow} 0 \quad \text { and } \quad\left|\gamma_{0}(t)-p\right| \rightarrow 0 \quad \text { as }|t| \rightarrow \infty \text {. }
$$

So if $|\varepsilon|<\varepsilon_{0} \ll 1$, (ii) of Theorem 3.1 is satisfied.

To prove (iii), let $x_{0}(t)$ be the $T$-periodic solution of (5), whose existence is shown in the remark of Theorem 2.1, and put $u_{0}(t)=x_{0}(\varepsilon t+\alpha(\varepsilon), \varepsilon)$.

Then $u_{0}(t)$ is a $(T / \varepsilon)$-periodic solution of system (11).

Denote $F(t, x)=f(x)+\varepsilon g(\varepsilon t+\alpha(\varepsilon), x, \varepsilon)$. 
Then $\mid F_{x}\left(t, v_{k}(t)\right)-F_{x}\left(t, u_{0}(t)\right) \rightarrow 0$ as $|t| \rightarrow+\infty$ uniformly with respect to $k$, because of

$$
\left|v_{k}(t)-u_{0}(t)\right|=\left|\gamma\left(\varepsilon t+a_{k} T+\alpha(\varepsilon), \varepsilon\right)-x_{0}\left(\varepsilon t+a_{k} T+\alpha(\varepsilon), \varepsilon\right)\right| \rightarrow 0 \quad \text { as }|t| \rightarrow+\infty .
$$

It follows from the roughness theorem that the variational equation

$$
\dot{x}=F_{x}\left(t, u_{0}(t)\right) x
$$

has an exponential dichotomy on $[0,+\infty)$, and being $F_{x}\left(t, u_{0}(t)\right)(T / \varepsilon)$-periodic, (18) has an exponential dichotomy on $\mathbb{R}$ and the corresponding to projection matrix function $Q(t)$ is $(T / \varepsilon)$-periodic.

If we put

$$
A_{k}(t)=F_{x}\left(t, v_{k}(t)\right), \quad B_{k}(t)=F_{x}\left(t, u_{0}(t)\right)-F_{x}\left(t, v_{k}(t)\right)
$$

then we may apply the roughness theorem to the following system

$$
\dot{x}=\left[A_{k}(t)+B_{k}(t)\right] x=F_{x}\left(t, u_{0}(t)\right) x .
$$

So, if $\tilde{P}_{k}(t)$ is the projection matrix function corresponding to the exponential dichotomy for

$$
\dot{x}=A_{k}(t) x,
$$

we have $\left|\tilde{\mathscr{P}}_{k}(t)-Q(t)\right| \rightarrow 0$ as $|t| \rightarrow+\infty$ uniformly with respect to $k$ because of $\left|B_{k}(t)\right| \rightarrow 0$ uniformly with respect to $k$.

We can notice that $\mathscr{P}_{k}(t)=\mathscr{P}_{k}(t-(2 k-1)(T / 2 \varepsilon))$, and that $\mathscr{P}_{k}(t), \tilde{\mathscr{P}}_{k}(t)$ and $Q(t)$ are uniquely determined, because the corresponding linear systems have an exponential dichotomy on $\mathbf{R}$.

Now

$$
\begin{aligned}
& \left|\mathscr{P}_{k-1}\left(t_{k-1}\right)-\mathscr{P}_{k}\left(t_{k-1}\right)\right|= \\
& =\left|\tilde{\mathscr{P}}_{k-1}\left(k \frac{T}{\varepsilon}-\frac{T}{\varepsilon}-2 k \frac{T}{2 \varepsilon}+3 \frac{T}{2 \varepsilon}\right)-\tilde{\mathscr{P}}_{k}\left(k \frac{T}{\varepsilon}-\frac{T}{\varepsilon}-2 k \frac{T}{2 \varepsilon}+\frac{T}{2 \varepsilon}\right)\right|= \\
& =\left|\tilde{\mathscr{P}}_{k-1}\left(\frac{T}{2 \varepsilon}\right)-\tilde{\mathscr{P}}_{k}\left(-\frac{T}{2 \varepsilon}\right)\right| \leqslant\left|\tilde{\mathscr{P}}_{k-1}\left(\frac{T}{2 \varepsilon}\right)-Q\left(\frac{T}{2 \varepsilon}\right)\right|+\left|Q\left(\frac{T}{2 \varepsilon}\right)-Q\left(-\frac{T}{2 \varepsilon}\right)\right|+ \\
& +\left|Q\left(-\frac{T}{2 \varepsilon}\right)-\widetilde{\mathscr{P}}_{k}\left(-\frac{T}{2 \varepsilon}\right)\right| \underset{\varepsilon \rightarrow 0^{+}}{\longrightarrow} 0 \text { uniformly with respect to } k \in \mathbb{Z} . \\
& t_{k}-t_{k-1}=\frac{T}{\varepsilon} \geqslant \tau \quad \text { if } \varepsilon \ll 1 .
\end{aligned}
$$

All the conditions of theorem 3.1 are verified, hence there exists a unique solution 
$y(t)$ of

$$
\dot{y}=f(y)+\varepsilon g\left(\varepsilon t+\alpha(\varepsilon)+\frac{T}{2}, y, \varepsilon\right)
$$

satisfying

$$
\sup _{t \in\left[t_{k-1}, t_{k}\right]}\left|y(t)-w_{k}(t)\right|<\varrho .
$$

Changing $t$ with $t+(2 k-1) T / 2 \varepsilon$ and writing $y_{a}$ instead of $y$ we have

$$
\dot{y}_{a}(t)=f\left(y_{a}(t)\right)+\varepsilon g\left(\varepsilon t+\alpha(\varepsilon), y_{a}(t), \varepsilon\right)
$$

and

$$
\sup _{-T / 2 \varepsilon \leqslant t \leqslant T / 2 \varepsilon}\left|y_{a}\left(t+(2 k-1) \frac{T}{2 \varepsilon}\right)-u\left(t+a_{k} \frac{T}{\varepsilon}\right)\right|<\varrho
$$

or putting $x_{a}(t)=y_{a}\left(\varepsilon^{-1}(t-\alpha(\varepsilon))\right)$ we have

$$
\varepsilon \dot{x}_{a}(t)=f\left(x_{a}(t)\right)+\varepsilon g\left(t, x_{a}(t), \varepsilon\right)
$$

and

$$
\sup _{-T / 2 \varepsilon+a(\varepsilon) \leqslant t \leqslant T / 2 \varepsilon \alpha(\varepsilon)}\left|x_{a}\left(t+(2 k-1) \frac{T}{2}\right)-\gamma\left(t+a_{k} T, \varepsilon\right)\right|<\varrho .
$$

Define now the function $\phi: \Sigma \rightarrow \mathrm{R}^{n}$, where $\Sigma$ is the set of the sequences $\left\{s_{k}\right\}_{k \in Z}$, putting $\phi(a)=x_{a}(0)$.

Proposition 3.1. - The function $\phi: \Sigma \rightarrow \mathbb{R}^{n}$, defined above, is a homeomorphism onto a compact subset of $\mathbb{R}^{n}$. it.

Proof. - The proof follows closely the one of Corollary 3.6 in [8], so we omit

Now we are able to prove the main theorem of this section:

Theorem 3.3. - Let $P: \mathbb{R}^{n} \rightarrow \mathbb{R}^{n}$ the Poincaré map of system (3).

Then the following diagram

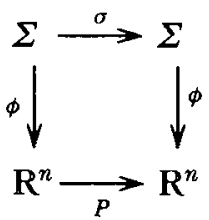


commutes. In other words for all $a \in \Sigma$ we have

$$
\left(P^{0} \phi\right)(a)=\left(\phi^{0} \sigma\right)(a) .
$$

Hence $P$ is isomorphic to the Bernoulli shift and then behaves chaotically.

ProOF. - Let $x_{0} \in \mathrm{R}^{n}$, and let $x\left(t, x_{0}\right)$ be the solution of

$$
\left\{\begin{array}{l}
\varepsilon \dot{x}=f(x)+\varepsilon g(t, x, \varepsilon), \\
x(o)=x_{0} .
\end{array}\right.
$$

The Poincaré map $P$ is defined by

$$
P x_{0}=x\left(T, x_{0}\right) .
$$

We want to prove that

$$
\left(P^{0} \phi\right)(a)=\left(\phi^{0} \sigma\right)(a)
$$

or that

$$
\phi(\sigma(a))=x(T, \phi(a))=x_{a}(T) .
$$

Put $x(t)=x_{a}(t+T)$. We find

$$
\varepsilon \dot{x}(t)=f(x(t))+\varepsilon g(t, x(t), \varepsilon)
$$

and

$$
\begin{array}{r}
\left|x\left(t+(2 k-1) \frac{T}{2}\right)-\gamma\left(t+a_{k+1} T, \varepsilon\right)\right|=\left|x_{a}\left(t+(2 k+1) \frac{T}{2}\right)-\gamma\left(t+a_{k+1} T, \varepsilon\right)\right|= \\
=\left|x_{a}\left(t+[2(k+1)-1] \frac{T}{2}\right)-\gamma\left(t+a_{k+1} T, \varepsilon\right)\right|<\varrho \\
\text { for } t \in\left[-\frac{T}{2}+\alpha(\varepsilon), \frac{T}{2}+\alpha(\varepsilon)\right] .
\end{array}
$$

By the uniqueness of $x_{a}(t)$ we have $x(t)=x_{\sigma(a)}(t)$ and so $x_{a}(T)=x(0)=x_{\sigma(a)}(0)=$ $=\phi(\sigma(a))$.

\section{REFERENCES}

[1] F. BATTELli - M. FeCKAN, Subharmonic solutions in singular systems, to appear in J. Differential Equations.

[2] F. Battelli - K. J. Palmer, Chaos in the Duffing equation, J. Differential Equations, 102 (2) (1993), pp. 276-301. 
[3] S.-N. Chow - J. K. HALE - J. Mallet-PARET, An example of bifurcation to homoclinic orbits, J. Differential Equations, 37 (1980), pp. 351-373.

[4] W. A. Coppel, Dichotomies in Stability Theory, Lecture Notes in Mathematics, 629, Springer-Verlag, Berlin (1978).

[5] M. FECKAN, Note on a local invertibility, to appear on Mathematica Slovaca.

[6] J. GuCkenheimer - P. Holmes, Non Linear Oscillations, Dynamical Systems and Bifurcations of Vector Fields, Springer-Verlag, New York (1983).

[7] U. KIRCHGRABER - D. STOFFER, Chaotic behavior in simple dynamical systems, SIAM Review, 32 (3) (1990), pp. 424-452.

[8] K. J. PALmER, Exponential dichotomie and transversal homoclinic points, J. Differential Equations, 55 (1984), pp. 225-256.

[9] S. Smale, Differentiable dynamical systems, Bull. Amer. Math. Soc., 73 (1967), pp. 747-817. 